reviews. The relevance of prions to biologicals, except those containing material of neural origin, is still unclear and not surprisingly no fully satisfactory recommendations are made for assuring their detection or removal.

The section on retroviruses contains useful reviews, especially on their inactivation in biological products, although this field has progressed significantly in the last 2 years. Sections 6 and 7 discuss the detection of viral contamination in laboratory animals, cell lines and biological products. The use of molecular biology based procedures such as PCR receives attention although the need for care in their application and interpretation is emphasised. The final session considers the regulatory aspects of viral safety in both conventional and high technology biological products. The general conclusions point towards the need for designing safety into the manufacturing process rather than attempting to remove or inactivate contaminating agents in the final product.

With the exception of the few chapters on subjects of interest to a wide audience, this volume will be useful mainly to those with a specialist interest in the viral contamination of biological products.

M. CORBEL

\section{Methicillin-Resistant Staphylococcus aureus. Clinical Management and Laboratory Aspects}

Edited by Mary T. Cafferkey. 1992. Marcel Dekker, New York. Pp. 202. £89.75.

The stated purpose of this book, one of a series on Infectious Disease and Therapy, is to review the clinical and laboratory aspects of the management of MRSA infection. The 12 chapters are concise and clearly presented with extensive references, although few are after 1990. Where there is repetition it usually benefits the reader. The topics are covered by authors from Ireland, the UK and the USA, and the book reflects work done in these countries, particularly in Dublin. The introductory chapter and chapter 2 cover the development of antibiotic resistance in Staphylococcus aureus and the evolution of MRSA. A useful contribution in chapter 4 discusses molecular techniques for strain identification; a greater focus on techniques such as pulse field gel electrophoresis would have been of benefit. This chapter also deals with the production of virulence determinants in S. aureus, noting that MRSA are more likely to produce enterotoxins than similar methicillin-sensitive strains. The use of phage typing is dealt with in a later chapter (chapter 7) on the epidemiology of MRSA. The biochemical and genetic basis of resistance and the expression of heterogeneous or homogeneous resistance (chapter 3) gives a background for subsequent chapters on detection of methicillin-resistant strains. Resistance correlates with presence of the mec gene, which codes for a new penicillin-binding protein, but other factors are also important in the expression of resistance and this subject is still not clearly understood.

A comprehensive chapter (chapter 5) looks at factors affecting laboratory detection of MRSA on which hospital control procedures depend. Reference is made to clumping factor- and protein A-negative strains of MRSA which would be missed without use of the tube coagulase test, and to difficulties with borderline resistance which may be due to hyperproduction of $\beta$-lactamase.

The argument over the clinical significance of MRSA and the distinction between colonisation and infection are comprehensively considered in chapter 6 , with the conclusion that MRSA cause a similar spectrum of disease to methicillin-sensitive strains. The contribution of risk factors including length of hospital stay and underlying disease in the patient are recognised.

The presence of multiple antibiotic resistance in many MRSA limits options for therapy. The choices and pitfalls are discussed in chapter 9 and elsewhere, stressing both the importance of antibiotic susceptibility testing before and during therapy, and the risk of resistance developing to several anti-staphylococcal agents to which MRSA may initially be sensitive. The value of vancomycin for successfu therapy is emphasised and the related glycopeptide, teicoplanin, is evaluated. Experience with antibiotic combinations is discussed. Although vancomycin resistance has not been described in $S$. aureus, the presence of transferable high-level resistance in enterococci underlines the importance of vigilance in testing for resistant strains. Two further chapters concentrate on the use of topical and systemic antimicrobial agents in attempts to eradicate carriage of MRSA. This relates to the final chapter which describes experience in the USA on the control of hospital MRSA. Application of the UK recommendations for control has been successful in the control of outbreaks, but experience shows that once endemic infection is established in a hospital, these procedures are much less successful in eliminating MRSA from the environment. Criticisms of the book's presentation are few, but genes such as mec should be in italic, figure 1 in chapter 7 did not reproduce well and there are some typographical errors.

Overall, the book achieves its stated aims in giving a comprehensive view of the clinical implications and therapy of MRSA and should be useful to clinicians and laboratory scientists looking for a background to the biological basis or clinical significance of MRSA. However, it is expensive at $£ 89.75 \mathrm{p}$ and is unlikely to be purchased by individuals, but it would make a very useful addition to the library.

S. Poston

\section{PCR in situ Hybridization. Protocols and Applications}

G. J. Nuovo. 1992. ISBN 0-88167-940-2. Raven Press, New York. Pp. 264. $\$ 94.00$.

This book describes the techniques whereby the extreme sensitivity of the polymerase chain reaction (PCR) is combined with the cell localising ability of in-situ hybridisation. Therefore, it is directed primarily towards histopathologists and virologists interested in localising viral DNA in tissue sections. It assumes the reader knows very little about DNA, PCR or in-situ hybridisation and cell morphology.

The first of nine chapters provides a brief introduction and is followed by chapters covering the basics of molecular biology and the PCR, both written in a simplified fashion. The fourth chapter describes the hot start modification of PCR. Then, to assist the researcher with the interpretation of in-situ hybridisation analysis, a brief discussion of the histological features of permanent tissues is provided. This is followed by a chapter on PCR in-situ hybridisation and chapters on its application to human papillomavirus and human immunodeficiency virus-1. Finally there is a chapter on RNA and mRNA reverse transcription PCR in-situ hybridisation.

Although some of the earlier part of the book may seem too basic for many readers, the later chapters are full of useful, well-presented protocols for those interested in the practical aspects of this technique. It is also nicely illustrated. For these reasons it is likely to be a useful introductory text for those wishing to acquire the technique of PCR in-situ hybridisation.

R. MATtheWS 